Wright State University

CORE Scholar

$12-2013$

\title{
Acceleration of Image Analyst Training with Transcranial Direct Current Stimulation
}

\author{
R. Andy McKinley \\ Lindsey McIntire \\ Nathaniel Bridges \\ Charles Goodyear \\ Michael Patrick Weisend \\ Wright State University - Main Campus, michael.weisend@wright.edu
}

Follow this and additional works at: https://corescholar.libraries.wright.edu/wsri

Part of the Neuroscience and Neurobiology Commons

\section{Repository Citation \\ McKinley, R. A., McIntire, L., Bridges, N., Goodyear, C., \& Weisend, M. P. (2013). Acceleration of Image Analyst Training with Transcranial Direct Current Stimulation. Behavioral Neuroscience, 127 (6), 936-946. https://corescholar.libraries.wright.edu/wsri/6}

This Article is brought to you for free and open access by the Wright State Research Institute at CORE Scholar. It has been accepted for inclusion in Wright State Research Institute Publications by an authorized administrator of CORE Scholar. For more information, please contact library-corescholar@wright.edu. 


\title{
Acceleration of Image Analyst Training With Transcranial Direct Current Stimulation
}

\author{
R. Andy McKinley, Air Force Research Laboratory, Wright-Patterson AFB, Ohio \\ Lindsey McIntire, Nathaniel Bridges, and Charles Goodyear, Infoscitex Corporation, Dayton, Ohio \\ Michael P. Weisend, Wright State Research Institute, Dayton, Ohio
}

Humans today are routinely and increasingly presented with vast quantities of data that challenge their capacity for efficient processing. To restore the balance between man and machine, it is worthwhile to explore new methods for enhancing or accelerating this capacity. This study was designed to investigate the efficacy of transcranial DC stimulation (tDCS) to reduce training time and increase proficiency in spatial recognition using a simulated synthetic aperture radar (SAR) task. Twenty-seven Air Force active duty members volunteered to participate in the study. Each participant was assigned to 1 of 3 stimulation groups and received two, 90-min training sessions on a target search and identification task using SAR imagery followed by a test. The tDCS anode was applied to site F10 according to the 10-20 electro-encephalographic electrode convention while the cathode was placed on the contralateral bicep. Group 1 received anodal tDCS at $2 \mathrm{~mA}$ for $30 \mathrm{~min}$ in the first training session and sham tDCS in the second session. Group 2 received the stimulation conditions in the opposite order. Group 3 did not receive stimulation at all. Results showed that participants receiving training plus tDCS attained visual search accuracies $\sim 25 \%$ higher than those provided with sham stimulation or no stimulation. However, a corresponding performance improvement was not found in the first training session for the change detection portion of the task. This indicates that experience with the imagery is important in the tDCS-elicited performance improvements in change detection.

Keywords: learning, noninvasive brain stimulation, cognitive performance, tDCS, military

The advent of the information age brought with it a daunting demand for increasing processing power, speed, and data storage. As technological advances continue at an accelerated rate to meet these demands, the information processing abilities of the human operator interacting with these computational systems has remained constant. That is to say the cognitive baseline of the operator (e.g., working memory capacity, speed of processing, andrate of learning) has not significantly changed. Consequently, the human is quickly becoming outpaced by information throughput and overwhelmed with the sheer volume of data generated by the systems they control. Resultantly, scientists and engineers are currently seeking cognitive enhancement technologies to help alleviate the mismatch between human limitations and computation system capabilities (Chief Scientist Air Force, 2010; Nelson, 2007; McKinley, Bridges, Walters, \& Nelson, 2012).

System automation has been one of the most popular solutions to improving efficiency and reducing cognitive workload on the human operator. However, there are some tasks that are not well suited for computer control given the current state of the artificial intelligence and computational logic. For example, computer automation cannot perform complex decision making regarding the appropriate use of lethal force or target recognition. In the latter, researchers have been trying to provide a reliable technology or methodology for automatic target recog- nition for over 20 years (Mishra, \& Mulgrew, 2010; Ahlberg, Klasen, Gronwall, Ulvklo, \& Jungert, 2003), but no approach currently exceeds human target recognition abilities. As a result, image analysis in Air Force intelligence, surveillance, and reconnaissance (ISR) missions remains a largely human endeavor. With the high demand for ISR in modern warfare, a large number of human analysts are needed to process the imagery gathered with airborne assets. In the face of exponentially increasing demand, it has become exceedingly difficult to train enough image analysts. As a potential remedy, we propose a change in the existing paradigm that incorporates technological advancements in neuroscience to improve learning and shorten training times.

One such rapidly developing neuroscience tool is transcranial DC stimulation (tDCS). Originally developed for treatment of neurological disorders such as Parkinson's disease, major depressive disorder, schizophrenia, stroke, dementia, chronic pain, and so forth, tDCS offers a method of augmenting neural activity in a way that may benefit human operator performance. The tDCS technology is simple, easy to 
apply, and relatively inexpensive, lending itself to application-oriented science. In particular, there is growing evidence that tDCS can enhance cognitive skills in healthy, normal humans. Decision making (Fecteau, Pascual-Leone et al., 2007; Fecteau, Knock, 2007), working memory (Fregni et al., 2005), implicit learning (Kinc- ses, Antal, Nitsche, Bártfai, \& Paulus, 2004), probabilistic learning (Hecht, Walsh, \& Lavidor, 2010), visual search (Bo- lognini, Fregni, Casati, Olgiati, \& Vallar, 2010), visuomotor coordination (Antal et al., 2004), language learning, (Flöel, Rösser, Michka, Knecht, \& Breitenstein, 2008), picture naming (Sparing, Dafotakis, Meister, Thirugnanasambandam, \& Fink, 2008), motor skill acquisition (Reis et al., 2009) are all improved with tDCS. For a complete review of these cognitive enhancement studies, see McKinley et al. (2012). Typically, tDCS uses a current regulator to provide between 1 and $2 \mathrm{~mA}$ of electric current between positive (anode) and negative electrodes (cathode) and into the brain. tDCS modifies neuronal resting potential; elevating neuronal excitability in brain regions effected by the anode and depressing excitability near the cathode (Paulus, 2003; Priori, 2003). For a more thorough description of the principles and physics related to tDCS, see Wagner, Valero-Cabre, and Pascual-Leone (2007).

To accelerate learning of specific objects and their subtle differences, it is important to identify the appropriate node(s) in the brain network that are active during training. Tanji and Hoshi (2008) describe the function of the ventral-lateral prefrontal cortex (VLPFC) and assert it is primarily involved with encoding and retrieval of all types of sensory information including visual (Tanji \& Hoshi, 2008). In addition, the VLPFC is active during selection and comparison of the visual information in support of decision-making processes related to object identification. Recently, Clark et al. (2012) attempted to accelerate learning of threat detection within simulated urban warfare scenes. They demonstrated that tDCS over the right VLPFC can significantly accelerate encoding and retrieval of threatening objects, providing initial evidence that perhaps military training could benefit from such an intervention. Falcone, Coffman, Clark and Parasuraman (2012) discovered these enhancements remain 24 hours after initial training. We attempted to expand on this research by evaluating the influence of tDCS on performance in other types of threat assessment tasks. In addition, we added a no-stimulation control group to gain a better understanding of the standard learning curve in military recruits.

Design

\section{Method}

This study utilized a one-factor mixed-subjects design. The factor evaluated was "group" (i.e., stimulation type) with the change in a' as the dependent variable. The factor "group" was tested between subjects and included three levels: participant group that received anodal stimulation ( $2 \mathrm{~mA}$ for $30 \mathrm{~min}$ ) in the first session and sham ( $2 \mathrm{~mA}$ for 30 seconds) in the second (AS), group that received sham stimulation in the first session and anodal stimulation in the second session (SA), and the group that received no stimulation at all in either session (Control). Study participants were randomly divided into these three groups. To test whether anodal tDCS facilitated learning when provided in the first training session, a one-way ANOVA was conducted using "group" as the factor and the change in a' from baseline to Test 1 as the dependent variable. Next, we were interested in whether anodal tDCS facilitated learning after some training had already been provided. To answer this question, we conducted a one-way ANOVA using "group" as the factor and the change in a' from Test 1 to Test 2 as the dependent variable. Finally, we tested whether the timing of the stimulation (i.e., provided early or late in the training) had an effect on the final performance levels. This was done using a one-way ANOVA with "group" as the factor and the change in a' from baseline to Test 2 as the dependent variable.

\section{Participants}

A total of 39 active-duty right-handed Air Force military members from Wright Patterson Air Force Base volunteered to participate in this study. Participants were dismissed if they had evidence of any of the following: a neurological diagnosis, a psychological diagnosis, psychological hospitalization, hospitalization for surgery/illness within 6 months of participation, taking of psychotropic medications, a shot in the left arm within 1 week of participation (i.e., flu, allergy, pain), nonremovable metal or tattoos around the head, uncorrectable vision impairments, pregnant or could become pregnant, smoking, treatment for drug/ alcohol within 6 months of participation, head injury within 30 days of participation, history of any of the 
following: learning difficulty, frequent headaches, attention deficit, severe head injury, seizures, fainting, migraine headaches, high blood pressure, diabetes, or heart disease. The participants were also screened for experience with analysis of Synthetic Aperture Radar imagery. Those that were not naïve to the task were not permitted to participate in the study. Of the 39 individuals that enrolled, 12 were dismissed because they met one or more of the study exclusion criteria. The remaining 27 ( 21 male, 6 female) were divided into three groups: anodal-sham $(n=9)$, sham-anodal $(n=10)$, and control $(n=8)$. All participants received $\$ 20 / \mathrm{hr}$ as compensation for their time if they participated in an off-duty status and a souvenir coin.

\section{Apparatus}

Transcranial DC stimulation was delivered with a MagStim (Whiteland, Wales, U.K.) DC stimulator. In place of the standard wet sponge electrodes that are delivered with the unit, custom silver/silver chloride electroencephalography (EEG) electrodes were utilized (see Figure 1). Both the anode and cathode each consisted of five of these EEG electrodes arranged in a circular pattern, as designed by the Mind Research Network (Petree et al., 2011). Each of the electrodes was $1.6 \mathrm{~cm}$ in diameter and was spaced $0.75 \mathrm{~cm}$ from the center and $0.1 \mathrm{~cm}$ apart as measured from the outer edge of the electrode to the outer edge of the neighboring electrodes to either side. With the $2 \mathrm{~mA}$ current divided evenly among the five electrodes, the estimated current density was $0.199 \mathrm{~mA} / \mathrm{cm}^{2}$. The anode was centered over F10 in the 10-20 EEG system, and the cathode was placed on the contralateral bicep. Electrodes were secured using medical bandages, and connectivity was ensured using highly conductive gel (SignaGel, Parker Laboratories, Fairfield, NJ).

To estimate the distribution of current in the brain, the electrode test configuration was evaluated in our finite element model. Only the head and neck were included in the anatomical data that was segmented to create the finite element model for visualizing cur- rent distributions. The extracephalic cathode was simulated with five electrodes arrayed across the skin at the back of the neck. To incorporate accurate geometry of the brain and inhomogeneities in the head model, the Finite Element Method (FEM) was employed to simulate the distribution of electric current due to tDCS (Bangera, 2008; Bangera et al., 2010). The following steps de- scribe the methodology to generate the highly realistic FEM model.

(1) Segmentation of the head volume: A combination of high- resolution PD (proton density) weighted and T1 weighted MRI from the subject was utilized in the segmentation process. The segmentation procedure was semiautomated and multistep. Automatic segmentation of gray matter, white matter, and subcortical structures was obtained from T1 images using Freesurfer (Dale, Fischl, \& Sereno, 1999; Fischl et al., 2002). Segmentation and labeling of the ventricles and subcortical structures was based on a statistical method (Fischl et al., 2002). The subcortical structures were lumped into one tissue type. The head volume was segmented into the 20 tissue types (15 unique materials): white matter (WM), gray matter (GM), subcortical, cerebrospinal fluid (CSF), cerebellum GM, cerebellum WM, ventricles, skull, diploe/spongy bone, fat, muscle, sinuses (frontal, sphenoid, ethmoid, and maxillary), air pockets near ear, sagittal sinus, spinal cord, teeth, optic chiasm, blood, eyes, and scalp. 


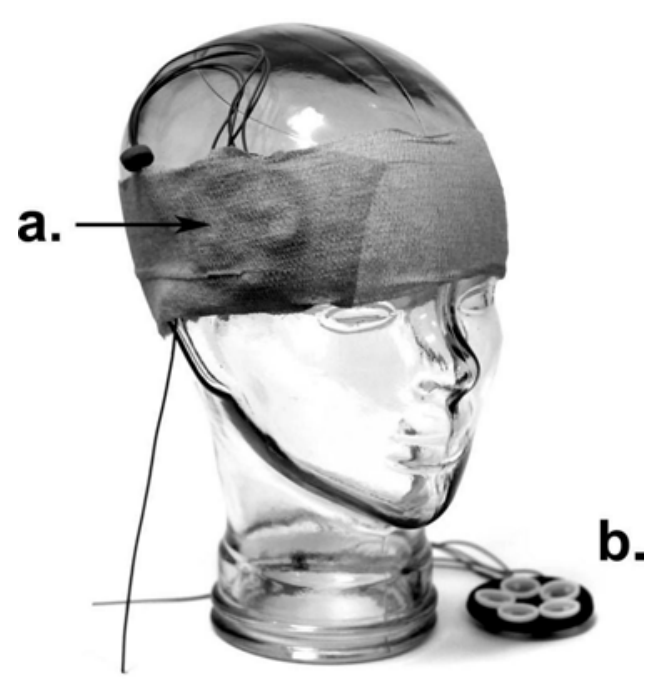

Figure 1. tDCS electrode configuration.

(2) Discretization into small elements from segmented volume: Tetrahedral elements were chosen to mesh the highly folded pattern of the brain due to its relative ease in representing complex geometries. AMIRA is used for mesh generation from the labeled and segmented head volume (Visualization Sciences Group, 2007).

(3) Solution using an efficient solver: ABAQUS, a suite of powerful engineering simulation programs, was used as the FEM solver (Dassault-Systèmes, 2007). ABAQUS also provided the ability to create realistic models of the stimulating electrodes used during tDCS. ABAQUS/Standard solved the Poisson's equation using the variational method to obtain an approximate solution for electrical potential and current density inside the head volume for a given tDCS configuration.

The estimated distribution of current flow using our new electrodes is depicted in Figure 2. The peak current concentrations were found in the posterior portion of the inferior frontal gyrus (i.e., VLPFC), right posterior lateral occipitotemporal gyrus, right uncus, right parahippocampal gyrus, and the right posterior orbitofrontal cortex. There is lesser but potentially effective current concentration in the left uncus, left posterior supraorbital cortex, and bilaterally medial occipital cortices.

\section{Stimuli: Synthetic Aperture Radar (SAR) Target Learning Task}

A visual search task was developed by the Mind Research Network and vetted through Image Analyst (IA) instructors from the Air Force's Air Education and Training Command (AETC) as a realistic representation of the training procedures employed for IA training. The task was developed with the Neurobehavioral Systems Presentation software (Albany, CA). It provided a circular red or blue reticle with which participants moved to identify targets in synthetic aperture radar (SAR) images of terrain with buildings and vehicles. There were a total of three different kinds of targets that could be present in the images including a T-62 tank, an SA-6 surfaceto-air missile launcher, and an SA-8 surface-to-air missile launcher. Each target could appear in six different orientations. The images were created by SAR imagery experts at Sandia National Laboratories in cooperation with MRN staff; thus, the locations, orientations, and numbers of targets in each image were known. All images came from the publically available MSTAR SAR database (http://cis.jhu.edu/data.sets/MSTAR/). The images were $1,200 \times 1,600$ pixels and the reticle was 160 pixels in diameter. Images were displayed on a standard 17-inch monitor with participants seated 32 in. from the screen (as measured from nasion to the center on the computer screen). In addition, the SAR images included distracters, vehicles that appeared similar to the target vehicles, to increase the difficulty of the task. The task was completed in five sessions; Baseline, Training 1, Test 1, Training 2, and Test 2 (see Figure 3). Baseline, Test 1, and Test 2 each consisted of 100 targets in approximately 50 images whereas the training sessions contained 200 targets in approximately $100 \mathrm{im}$ - ages. There were 1, 2, or 3 targets per image. Each test required 18 


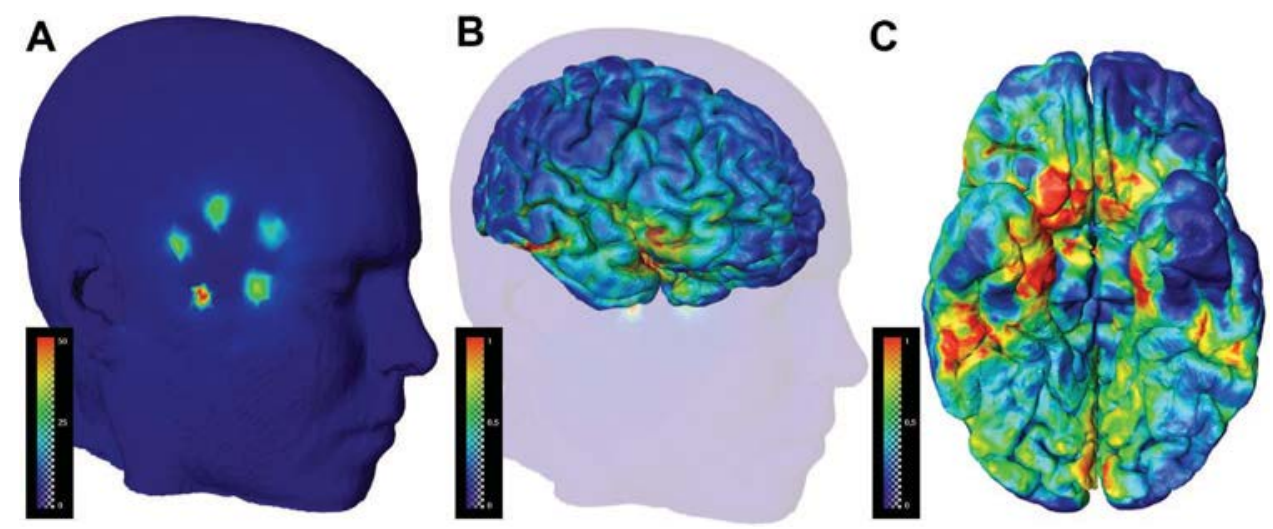

Figure 2. Finite element model of estimated current distribution in the brain.

to 30 minutes depending on the speed of the subjects' responses. Training 1 and Training 2 consisted of two sessions of 200 targets presented in approximately 100 images. All responses were made using a mouse in the right hand. Again, there were 1, 2, or 3 targets per image. Each training session lasted approximately 90 minutes.

During the three testing sessions, Baseline, Test 1, and Test 2, participants moved the reticle until a candidate target was encircled and clicked the left button to mark it. With the mouse click potential labels for the image appeared around the reticle. After the image label was selected the reticle returned to the center of the screen and left the previously selected target marked with a circle. The participant could then make additional selections. The participant continued to mark targets until all candidate targets were marked to their satisfaction. At this point the participant clicked the mouse button in the center of the screen and selected next to begin the change detection part of the trial. During change detection, the reticle appeared in the center of the screen. The participant scanned the previous target locations for the addition, deletion, rotation, movement, or exchange of a target vehicle. Moving the reticle over the target location and clicking the mouse button marked the changed item. Each of the four types of change (addition/deletion, rotation, movement, and exchange of a target vehicle) was equally probable. There were six possible orientations, each in 60-degree increments relative to north (i.e., $0,60,120,180,240,300$, and 360). In the case of movement, the possible new positions were equally distributed into the four quadrants of the screen. Additionally, there was always exactly one change per trial. If during any part of the trial, the participant made no selections for 15 seconds, the next image or phase of the trial was presented. 
The Sequence of training and testing blocks with notations for Active and Sham TDCS.
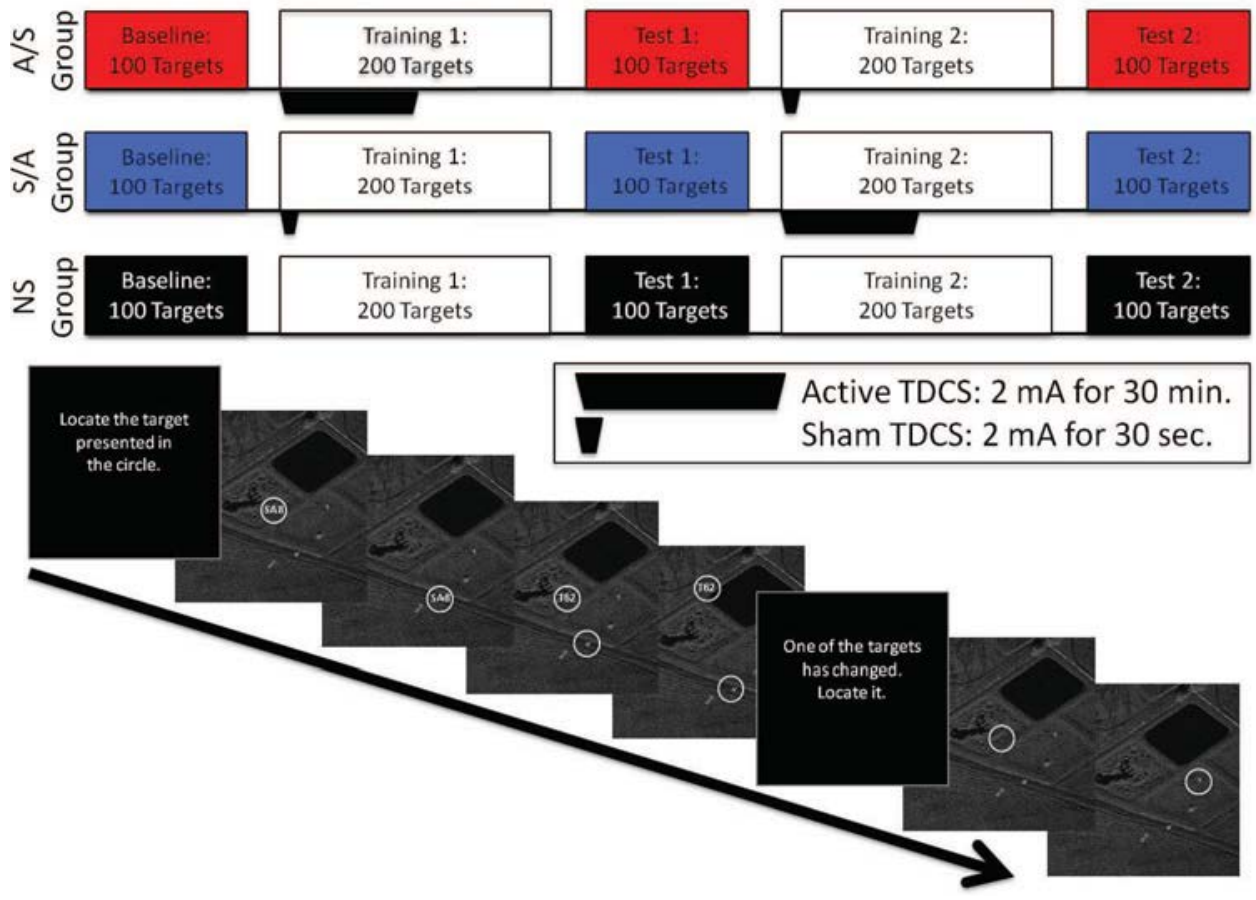

Figure 3. Synthetic aperture radar task training and testing sessions. 
During the training sessions, Training 1 and Training 2, the target items and response requirements were identical to the testing sessions. However, unlike the testing sessions, the software delivered feedback about the participants' target choices. When an incorrect selection was made, a red $X$ appeared in the reticle to indicate that the item in the reticle was not a target of interest. After the red $X$ was presented, the reticle would return to the center of the screen so the subject could make additional selections. Participants continued making selections until the correct target was selected or the 15-s time limit was reached. When all the targets had been correctly marked, or the maximum time of 15 seconds had been reached, the image would be presented for an additional 15 seconds with the incorrect selections surrounded by a red circle and the correct targets labeled and highlighted with a yellow circle. This provided feedback to the participant and permitted them time to study the image and targets. At the end of each trial, the participant was prompted to find something that had changed in the image. The same image would then be presented to the participant with one of the targets modified in some fashion. Possible changes included a target moving to a new location, a target rotating into a new orientation, the removal of a target, or the target changing from one target type to another (i.e., a T-62 replaced with an SA-6). Again, participants controlled a reticle and depressed the left mouse button when it encircled the target that had been changed. As before, a red $X$ would appear if the selection was incorrect and the reticle would return to the center of the screen. Participants continued making selections until the correct target was selected or the 15-s time limit was reached. As in the "target selection" portion of the task, participants were provided with a feedback screen for 15 seconds with the correct and incorrect selections highlighted.

From this task we extracted true positives (correct identification of targets), false positives (incorrect identification of a nontarget or terrain feature as a target), and false negatives (incorrectly asserting that there are no targets or never finding the target within the time limit) in the SAR images. Because the task never presents an image that does not contain a target, there are no true negatives (correctlyasserting there is no target within the image). As a result, the traditional sensitivity analyses ( $\left.d^{\prime}\right)$ could not be completed. The time to complete identification of targets in each image was recorded to $10^{-5}$ seconds; the default precision in the Neurobehavioral Systems Presentation software.

\section{Procedure}

All experimental procedures used in the protocol were reviewed by the Wright-Patterson Air Force Base Institutional Review Board (IRB). Subjects first provided written informed consent to participate before being registered into the study or filling out Initial Screening Questionnaires. These questionnaires gathered information regarding their fitness for participation and back-ground. If the participant failed to meet the inclusion criteria, they were excluded from participation and no identifiable data was retained. Those that did meet the inclusion criteria completed a neuropsychological test including a shortened Wechsler Abbreviated Scale of Intelligence and a NEO-Five Factor Inventory (NEO- FFI) personality test administered by a trained research associate. The behavioral battery consists of measures of individual differences comprising intellectual, personality, working memory, speed of processing and problem solving ability. This testing lasted approximately 1 hour. Afterward, subjects were given a short break followed by a short movie clip describing the SAR task instructions and tDCS procedures. Next, subjects performed a SAR task test that provided a baseline measure of their performance before the subject participated in any training activities.

After the baseline test, participants were asked to complete a mood questionnaire. Then the participants were assigned to groups; anodal tDCS ( $2 \mathrm{~mA}$ for $30 \mathrm{~min}$ ) during training session 1 and sham ( $2 \mathrm{~mA}$ for $30 \mathrm{sec}$ ) during training session 2 (AS) or, the opposite order, sham during training session 1 and anodal tDCS during training session 2 (SA). Following group assignment, the tDCS electrodes were affixed to the scalp and the training/tDCS paradigms began. A third group of participants, the control group, did not receive tDCS and did not wear the electrodes. The research technicians were blind to the tDCS condition by a coded input to the stimulator. A few seconds after beginning tDCS, the participants completed a sensation questionnaire that asked them to rate sensations of itchiness, heat, pain, and overall discomfort on an 11-pt scale (0 indicating no feeling at all, 10 indicating extreme sensation). Next, the participants completed the training sessions of the 
SAR task lasting approximately 90 minutes in total. Five minutes after the start of the training, the task would be paused and the participant would again fill out the sensation questionnaire. If at any time a sensation rating of 7 or greater was reported, the tDCS was immediately turned off and study procedures were terminated. The subject was evaluated by the medical monitor and released. Those in the control group also filled out the sensation questionnaires, even though stimulation was not present. Following the four training sessions a nofeedback test was completed. Lastly, they completed the mood questionnaire again to ensure there were no lingering effects of the stimulation. This indicated the conclusion of the first session.

After the first session, participants were given a 30-min break. They then repeated the procedures for Session 1, with the opposite stimulation condition. Before leaving, the subjects were required to complete an Exit Questionnaire to get feedback on their experience participating in the study and a subjective opinion about which condition they felt they received anodal tDCS.

\section{Results}

Because all of the images contained at least one target, there were no trials that would result in the correct assertion that a target did not exist (i.e., True Negatives [T-]). For this reason, it is not possible to calculate the traditional operator sensitivity measure, known as $d^{\prime}$ (Sorkin, 1999). Consequently, a measure known as a' was used as the dependent variable of interest. This is defined as the ratio of all correct target selections (true positives $[\mathrm{T+}]$ ) to the sum of all possible responses including $T+$, false positives (F+) (i.e., incorrect target selections/selecting a nontarget as a target), and false negatives (F-) (i.e., incorrectly indicating a target was not present). Performance was compared between participants that received anodal stimulation during Training 1 then sham during Training 2 (AS group), participants that received sham during Training 1 then anodal stimulation during Training 2 (SA group), and those that received no stimulation (control group) for both the target search and change detection portions of the task.

\section{Target Search}

Because the stimulation condition changed after the first session for the two stimulation groups, the analysis was segregated into three parts with a separate analysis of variance (ANOVA) for each segment. The first one-way ANOVA tested whether there was a significant effect of "group" (i.e., stimulation type) from baseline to Test 1 with group (AS, SA, or control) as a between-subjects variable and the change in a' as the dependent variable. The results showed a significant effect of "group", $F(2,24)=6.07, p=.0074$ on $a^{\prime}$. The means in change of a' from the ground target search portion of the SAR task are presented in Figure 4. For pairwise comparisons of the means within the session test, $t$ tests (pooled error) were conducted with an alpha of 0.05 . Importantly, these values are the means of the change in a' from one test to another, rather than the mean of a' at a specific test. The mean change in a' from the AS group $(0.548 \pm 0.035)$ was significantly higher from baseline to Test 1 than the control group $(0.295 \pm 0.070)(p=.0021)$. The difference in the mean change in a' from baseline to Test 1 in the SA group $(0.455 \pm 0.045)$ was also significantly higher than that of the control group $(p=.0346)$. There was no difference in the change of a' between the AS and SA group from baseline to Test 1.

The second one-way ANOVA tested whether there was a significant effect of "group" (i.e., stimulation type) from Test 1 to Test 2 with group (AS, SA, or control) as a between-subjects variable and the change in $a^{\prime}$ as the dependent variable. The results revealed a significant main effect of "group" on $a^{\prime}, F(2,24)=5.45, p$ $=.011$. Again $t$ tests with pooled error were used to examine differences in the mean change in a'. The change in a' of the AS group $(0.446 \pm 0.037)$ was not statistically different than the mean performance of the SA group $(0.630 \pm 0.040)(p=.064)$, although it was approaching significance. The effect size as measured by Cohen's $d$ for this comparison was a rather high 1.64. Often, values over 0.8 indicate a strong effect; hence we believe that the comparison would be statistically significant with more subjects. Further, the mean change in $a^{\prime}$ for the SA group $(0.097 \pm 0.023)$ was significantly higher than the control group $(0.001 \pm 0.038)$ from Test 1 
to Test $2(p=.021)$, but the mean for the AS group $(-0.018 \pm 0.020)$ was not $(p=.63)$.

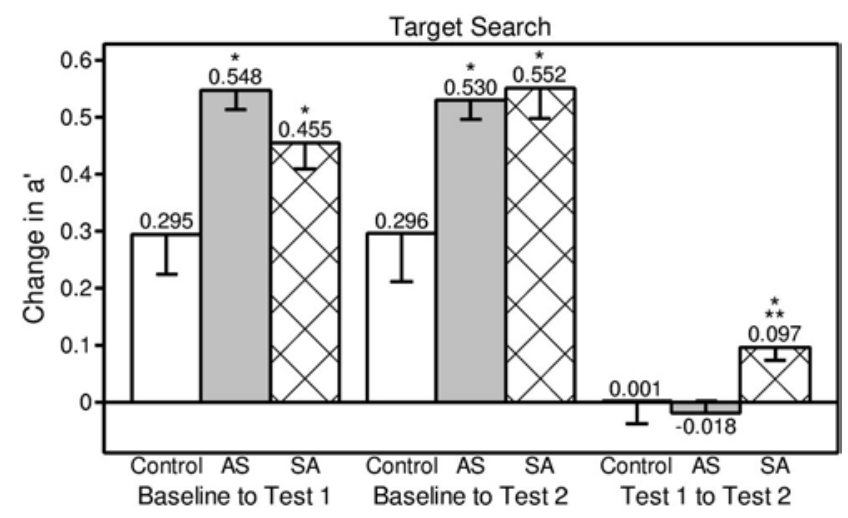

Figure 4. Target search change in a' means.

The third one-way ANOVA tested whether there was a significant effect of "group" from baseline to Test 2 with group (AS, SA, or control) as a between-subjects variable and the change in a' as the dependent variable. Again, the results showed a significant effect of group on the outcome measure, $F(2,24)=5.36$, $p=.012$. Paired $t$ tests with pooled error showed that the both the AS group $(0.446 \pm 0.037)$ and the SA group $(0.552 \pm 0.037)$ had a significantly higher change in a' when compared to the control $(0.296 \pm$ $0.086 ; p=.0125 \& p=.0059$, respectively). The comparison of mean change in a' between the AS and SA group was not significant.

A separate analysis was conducted to examine differences in a' at the testing sessions (means shown in Figure 5). A two by three ANOVA was conducted with factors "test" and "group". "Test" was analyzed within subjects with two levels (Test 1 and Test 2). "Group" was tested between subjects with three levels (control, $\mathrm{SA}$, and AS). There was a significant interaction between "test" and "group", $F(2,24)=5.45, p=.011$. For pairwise comparisons of the means within the session test, $t$ tests (pooled error) using the means for the participants from that session only were conducted with an alpha of 0.05 . The mean accuracy of the AS group $(0.732 \pm 0.032)$ was significantly higher in Test 1 than the control group $(0.573 \pm 0.038)(p=.0118)$. The difference in the mean of the SA group $(0.625 \pm 0.046)$ and that of the AS group approached significance $(p=$ .0638). The effect size as measured by Cohen's $d$ was 0.91 for this comparison. The high Cohen's $d$ indicates there is likely an effect of the stimulation between the SA and AS groups and that the comparison would be statistically significant with more subjects. The accuracy means for the control group and SA group did not differ significantly in Test 1.

In Test 2 , the mean a' of the AS group $(0.714 \pm 0.035)$ was not statistically different than the mean performance of the SA group $(0.721 \pm 0.054)(p=.90)$. However, the mean a' for both the AS and SA group was significantly higher than the control group $(0.574 \pm 0.036)$ in Test $2(p=.041$ and $p=.028$, respectively). As a percentage, the mean a' for the AS and SA groups were $24.3 \%$ and $25.6 \%$ higher than the mean a' for the control group in Test 2.

\section{Change Detection}

The analyses described in the Target Search section were repeated for the "change detection" portion of the task. The change in a' means from each group (control, AS, and SA) and test (baseline, Test 1, and Test 2) are presented in Figure 6. As in the visual search portion of the SAR task, the first one-way ANOVA tested whether there was a significant effect of "group" (i.e., stimulation type) from baseline to Test 1 with group (AS, SA, or control) as a between-subjects variable and the change in a' as the dependent variable. The results showed there was no effect of "group", $F(2,24)=1.26, p=.3$.

The second ANOVA tested whether there was an effect of "group" on the change in a' from Test 1 to Test 2. While the results did not show a significant main effect of "group" on the change in a', the analysis suggests the test was approaching significance, $F(2,24)=3.08, p=.065$. Hence, an effect may exist, but 
was likely masked due to the low $n$ size.

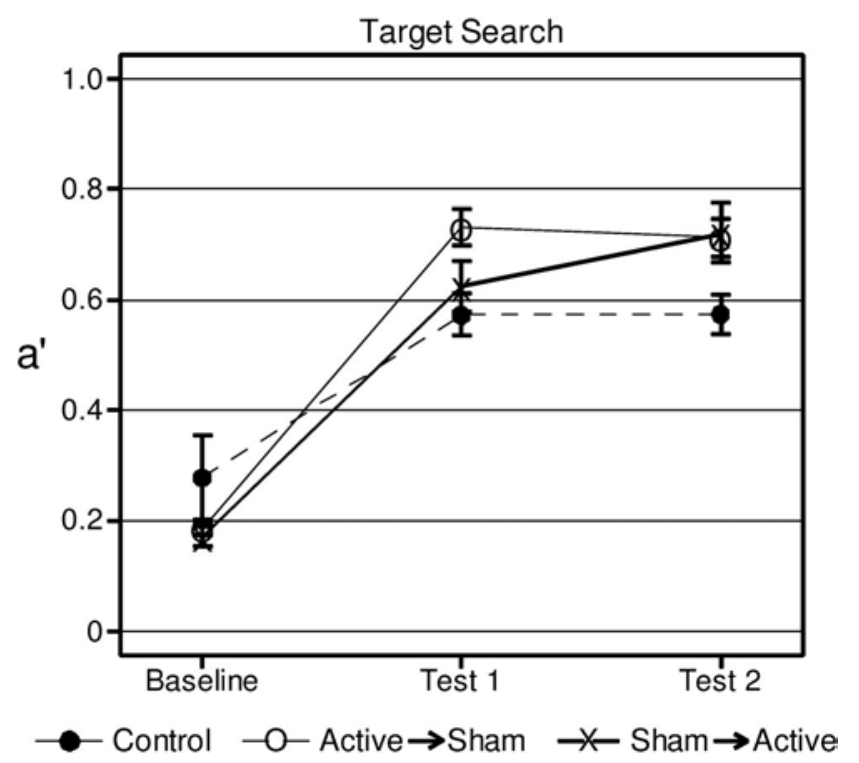

Figure 5. Target search accuracy means $\left(a^{\prime}\right)$.

The final ANOVA suggests there is a main effect of "group", $F(2,24)=4.89, p=.016$ on the change in a' from baseline to Test 2. For pairwise comparisons of the means within each session test, $t$ tests with pooled error were performed. As seen in Figure 6, a significant difference was found between SA group and both the AS and control groups. The mean change in a' of the SA group $(0.630+0.040)$ was statistically higher than the mean performance of the control group $(0.329+0.118)(p=.0406)$. In the comparison between the AS and SA group, the variance of subjects for the control group was significantly greater than either the AS or SA groups ( $p \leq .05)$. This was due in large part to two subjects, both having a relatively high baseline a' (approximately 0.6 ) and changes from baseline to Test 2 of 0.06 and -0.31 . Removing the subject with a -0.31 change had little effect on the $F$ test of group ( $p=.0165$ with this subject, $p=.0169$ without); however, the paired comparison of AS versus SA was greatly influenced ( $p=.0637$ with this subject, $p=.0168$ without). With this in mind, paired tests within baseline to Test 2 for change detection used $t$ tests, which don't use pooled error. The resulting $t$ test showed the change in a' for the SA group was significantly higher than that of the AS group ( $p=.0035)$.

As with the target search portion of the task, a two by three ANOVA (mixed design) was conducted with factors "test" and "group" to examine differences in a' at the testing sessions. The a' means for the change detection task are shown in Figure 7. The results suggest a significant main effect of "test" on a', $F(2,24)=$ 28.31, $p=.0001$. Again, $t$ tests (pooled error) were utilized to examine pairwise comparisons in the means. All three groups exhibited significantly higher a' values in Test 1 when compared to baseline (AS, $p=.0001$; SA, $p=.0001$; and control, $p=.024)$. Additionally, they all improved significantly in Test 2 when com-pared to baseline, (AS, $p=.0001 ; \mathrm{SA}, p=.0001$, and control, $p=.027$ ). Importantly, the SA group was the only one to show a statistically significant improvement in accuracy from Test 1 to Test $2(p=.00012)$.

\section{Discussion}

Our study examined the effects of tDCS on learning of targets within static images in an effort investigate tools that may accelerate object recognition. By receiving performance feedback during training, participants could identify their mistakes and study the SAR images to examine the correct targets more thoroughly. Through the training, it was expected that performance in target search would improve, but the effects of the tDCS on this learning curve were not known. Our results showed that without stimulation, the ability of the participants to correctly identify targets as measured by accuracy (a') elevated significantly in the first session. A comparable increase in the target search portion of the task was not found in the second 
training session, indicating their learning rate asymptotically stabilized and there was a diminishing return on the training time investment. Examining the relative change in performance between groups from baseline to Test 1, we found that both the stimulated group (AS) and sham group (SA) exhibited a significantly greater improvement when compared to

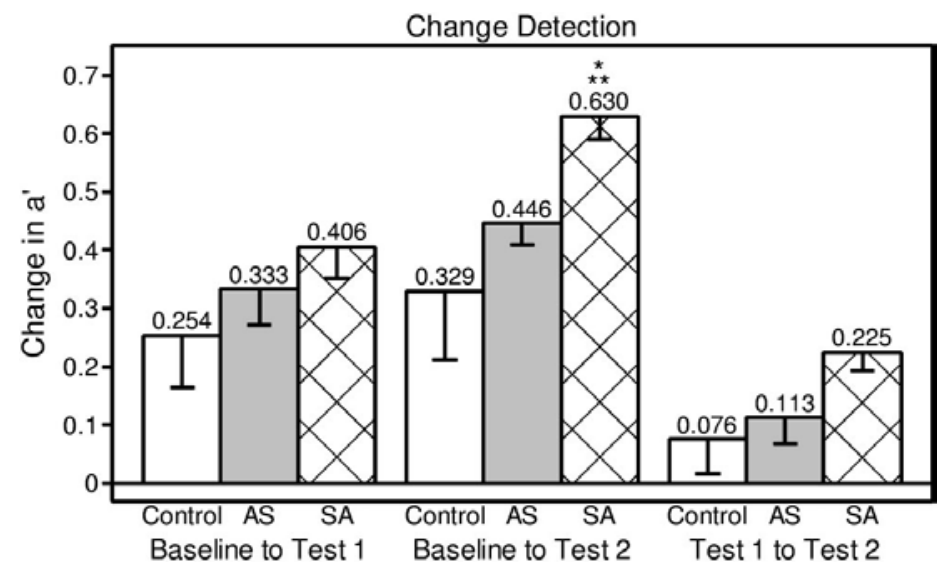

Figure 6. Change detection task change in a' means.

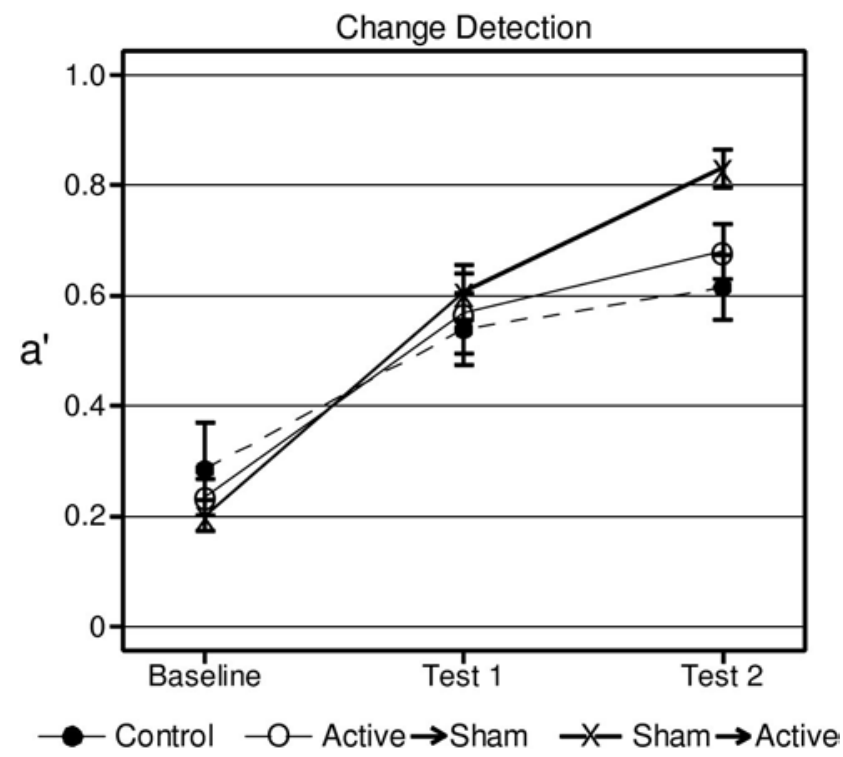

Figure 7. Change detection accuracy means (a').

the nonstimulated group (control). However, inspection of the mean performance achieved at Test 1 (i.e., end result of training Session 1), shows the control group's performance was not statistically different than the SA group (see Figure 5). Examining the data, the difference in the slope of the learning curve appears to be driven by the relatively higher mean baseline in the control group when compared to the AS and SA groups. While the comparison of the learning slopes (i.e., change in a') between the AS and SA group also did not achieve statistical significance, the test was approaching significance. This coupled with the high effect size denoted by Cohen's $d$ show that this effect would likely be significant with a slightly higher $n$ size. Furthermore, the AS performed significantly better than the control group at Test 1 . Taken together, we can infer that the stimulated participants learned significantly more objects while undergoing the same training as their nonstimulated counterparts.

In session 2, we reversed the stimulation conditions, applying anodal tDCS to the SA group and sham tDCS to the AS group. With no additional stimulation in session 2, the AS group did not exhibit further performance improvements, which indicates that additional training in the absence of anodal tDCS did not significantly improve learning. However, the SA group exhibited a performance improvement that approached significance while undergoing anodal tDCS in session 2. Given the large Cohen's $d$, we believe there is an effect 
that was masked by a small sample size. Importantly, a similar increase was not found in the control or the AS groups, both of which did not receive anodal tDCS. When comparing the means at Test 2, the SA group's accuracy (a') was at the same level as the AS group, which had previously outpaced the SA group by approximately $25 \%$. This indicated that stimulation either early in the training or late in the training had beneficial effects on object learning. In fact, both groups that received anodal stimulation at some point during their training (AS and SA) were performing at accuracy levels approximately $25 \%$ higher at the end of session 2 than that of the participants that did not receive any stimulation during their training. A recent study suggested that tDCS applied early in the training (i.e., from novice to intermediate stage) had a larger effect on performance than when applied later (i.e., from intermediate to expert stage) (Bullard et al., 2011). While our data do not support this conclusion, it is possible that our subjects never attained performance that would be considered "expert". Hence, the participants' reported herein may have still been in the "early" phase of learning which suggests that beneficial tDCS effects on learning would diminish with greater expertise. Additional studies would be required to validate this hypothesis. Furthermore, because institutional review boards have not yet approved more than 30 min of tDCS in healthy participants per day, it is not known if tDCS applied in both training sessions would yield a greater improvement in performance when com- pared to tDCS in just one of the sessions. We believe that performance would eventually achieve an asymptote, but additional data is needed to verify its existence and then decipher the amount of tDCS required to reach it.

The results from the change detection portion of the task illustrated that there was no difference in performance between any of the study groups following session 1 . Though all subjects showed a significant improvement from baseline to test two-regardless of stimulation condition, the SA group showed significantly greater improvement. This suggests that tDCS only facilitated learning of the recognition of changes in the targets when they had some experience with the imagery. In other words, the neural changes associated with mastery of the task were not possible until subjects reached a certain level of proficiency. Coffman et al. (2012) found similar results noting that tDCS had a much higher effect on object detection for images that were repeated than for novel test images. The authors concluded that this phenomenon may have been caused by tDCSinduced plasticity in brain networks involved with image encoding. Hence, repeated stimuli would generate a larger catalog of remembered images to aid in the detection of changes within the image. Expressed colloquially, the participants needed to first learn what the targets were before they could accurately identify if one had changed in some manner. It is for this reason that we believe tDCS effects interacted with experience in the change detection task and not the target search task. Nevertheless, Test 2 results provide evidence that tDCS can significantly accelerate the learning process.

The sensation values from the stimulation were low with an overall mean value of $1.22 \pm 0.25$, which is lower than corresponding studies with sponge electrodes, and subjects were more effectively blinded to the stimulation condition. The increased sensation ratings by the SA group suggest that when given a reference point of no stimulation, subjects are more aware of the active condition. Although $60 \%$ responded correctly when asked which of the two sessions they believed they received anodal stimulation, this result is only slightly above random chance. Hence, it could be that they simply achieved a correct response through chance alone. This assumption is further supported by the objective performance data. There were no statistical differences in task performance between the group receiving sham stimulation (SA) and group receiving no stimulation (control) in session 1. This indicates that there was no "placebo effect" in the sham group resulting from the transient skin sensations such as tingling, itchiness, or warming typically experienced for the first minute of stimulation or from the participants' possible expectation of improved performance from stimulation. Nevertheless, there is still work to be done to perfect the sham condition.

Previous work has documented both the acceleration of training and the enhancement of performance with tDCS. Straightforward targeting of the motor and visual cortices facilitates motor learning (Nitsche et al., 2003), visuomotor coordination (Antal et al., 2004), and decreases reaction times (RTs) (Nitsche et al., 2003). Similarly, cognitive neuroscience has led to targeting the prefrontal cortex to facilitate working memory (Berryhill \& Jones, 2012; Fregni et al., 2005; Gladwin, den Uyl, \& Wiers, 2012; Teo, Hoy, Daskalakis, \& Fitzgerald, 2011; Mulquiney, Hoy, Daskalakis, \& Fitzgerald, 2011). Not so intuitively, prefrontal TDCS has also been reported to speed decision-making toward high-probability events in a probabilistic decision task 
(Hecht et al., 2010), decrease RT in an implicit associations test (Gladwin, den Uyl, Fregni, \& Wiers, 2012), facilitate learned inhibition (Ditye, Jacobson, Walsh, \& Lavidor, 2012), impair episodic verbal learning (Elmer, Burkard, Renz, Meyer, \& Jancke, 2009), facilitate classification learning when the prefrontal cortex is targeted (Kincses et al., 2004), alter the recall of unpleasant and pleasant pictures (Penolazzi et al., 2010), and decrease risk-taking behavior in a gambling task (Fecteau, Knock et al., 2007). It is not clear how these tasks are related to prefrontal function except that working memory may be an important component of these tasks.

The stimulation in the experiments reported herein was also prefrontal with two important differences. First, our anode was placed near F10, lateral, anterior, and inferior to the common F3 or F4 frontal placements in the papers cited above. Because of its location, the cortical stimulation likely influenced the temporal lobe as well as the frontal lobe. Further, models of the F3 or F4 placements have not shown significant temporal lobe stimulation (Sadleir, Vannorsdall, Schretlen, \& Gordon, 2010; Datta et al., 2009). Previous studies show that temporal stimulation facilitates visual learning (Chi, Fregni, \& Snyder, 2010), facilitates the recall of name/face associations (Ross, McCoy, Wolk, Coslett, \& Olson, 2010), and facilitates solving insight based problems (Chi \& Snyder, 2011). The second important difference in our studies is the use of an extracephalic cathode. The cathode, sometimes called the reference or return, was placed somewhere on the scalp in all of the studies from other groups, cited above. This is a potential confound since the cathode, typically thought to inhibit the underlying cortex, produces cognitive effects (Kincses et al., 2004; Ambrus, Antal, \& Paulus, 2011; Hammer, Mohammadi, Schmicker, Saliger, \& Munte, 2011; Penolazzi et al., 2010). The data reported herein are more straightforwardly reportable as the effects being the result of anodal stimulation. This extracephalic cathode approach shows consistent, replicable results across multiple studies in our lab (Bullard et al., 2011; Clark et al., 2012; Coffman et al., 2012) and others (Falcone et al., 2012).

While the mechanisms responsible for the learning improvements found here cannot be derived from the data collected, theories exist that may help explain these phenomena. One possibility is that the tDCS current elevated excitability in the VLPFC, which is involved in encoding and retrieving visual information regarding the recognition of an object. By pairing the continued elevated activity in this area with the visual stimuli, weak synapses related to encoding and retrieving visual objects may have been strengthened, similar to the classical conditioning paradigm. Alternatively, sustainment of the elevated activity during the training may have served to facilitate activity of the natural reinforcement system via dopamenergic pathways. Because participants received feedback on their performance following stimulus presentation, it is reasonable to suspect that their reinforcement system would activate (i.e., their brain signaled something good happened when correct selections were made). Mirenowicz and Schultz $(1994,1996)$ showed that the reinforcement stimulus is activated primarily by novel stimuli. Once the participant becomes familiar with the task, the reinforcement system delivers less dopamine to rein-force encoding of the new information. The dopamine release is signaled by the ventral tegmental area, which has projections to the prefrontal cortex (Carlson, 2010). Hence, the higher excitability in VLPFC caused by the tDCS may have further stimulated release of dopamine, thereby preventing the natural decline of the reinforcement system. This, in turn, would theoretically further strengthen neural connections associated with encoding the object identity information.

Recently, we found the tDCS over the dorsolateral prefrontal cortex (DLPFC) significantly improved performance on a sustained attention (vigilance) task (Nelson, McKinley, Golob, \& Warm, 2013; McKinley, Nelson, \& McIntire, 2010). Because there are many stimuli in the environment completing for attention, information that is not attended will likely not be encoded or retained (Desimone, 1996). Given that tDCS is not particularly focal (Wagner, Valero-Cabre, \& Pascual-Leone, 2007), brain areas close to the target site may also receive substantial amounts of current (Sadleir et al., 2010). As a result, it may be the case that participants may have received stimulation in the DLPFC as well, which caused improvements in attention. Thus, the improvements in learning could have been a direct result of attending to more of the visual stimuli during training.

Another possibility is that the learning improvements observed in this experiment may have been caused by a Hebbian effect due to the increased cortical activity induced through tDCS. Colloquially summarized, Hebbian synaptic plasticity can be stated as "cells that fire together wire together." This Hebbian 
mechanism appears to be important in the effect of tDCS on enhanced eyeblink conditioning in the rabbit (Márquez-Ruiz et al., 2012). Further, tDCS enhances BDNF dependent synaptic plasticity (Fritsch et al., 2010). In the present study, tDCS during training produced a lasting effect on performance 30-90 minutes after stimulation. This effect endures for at least 24 hours (Falcone et al., 2012). Other work in this laboratory suggests that the effects of tDCS on excitability return to baseline by $40 \mathrm{~min}$ poststimulation. Thus, enhanced Hebbian plasticity at least at the 90-min and 24-hr tests could be important in the mechanism of the findings reported herein.

The complexity of the task used in this experiment undoubtedly involves a relatively large brain network. We have not performed cognitive experiments involving control tasks to disambiguate the specific effects on performance. One control task that has been evaluated is the stimulation of alternative sites with tDCS. Clark et al., (2012) did not find a significant effect when using alternative electrode locations and current strengths in a similar threat detection task. The effect is also not equal for all stimuli presented. Coffman et al. (2012) show the effect is different for novel and repeated stimuli. Additionally, McKinley et al. (2010) showed that tDCS of frontal cortex improved sustained attention. Taken together, one potential hypothesis is that enhanced vigilance results in greater time on task and thus improved encoding for specific objects presented visually.

When discussing neuromodulation in healthy individuals to improve cognitive skills, comments on ethical concerns are certainly warranted. In fact, a recent article in Wired magazine provided a commentary that cautioned the neuroscience community to carefully consider ethics and human safety when applying such tools in military contexts (Scott, 2012). As we previously reported in McKinley et al. (2012), we certainly agree that such research should proceed with care and that all efforts to protect the safety of the military operators should be employed. Because critical missions such as image analysis and target detection are essential to preserving lives of fellow soldiers and quite possibly civilians, we submit that interventions such as tDCS should be considered as they may become appropriate and indispensable tools when applied in a manner conducive to the safety and well-being of the operator.

\section{Conclusions}

Regardless of the mechanism, the results presented in this study provide additional data that support earlier findings of Clark et al. (2012) and Falcone et al. (2012), suggesting tDCS is a valid catalyst for accelerating learning of threat detection. These data also indicate that such results are not task specific and are robust across tasks testing similar cognitive functions. We have also provided new evidence that tDCS given during training greatly enhances target acquisition accuracy over individuals that did not receive any stimulation. Lastly, this study has shown that change detection accuracy is not affected by tDCS until participants be-come familiar with the task. It should be noted that these results were derived from a laboratory, and additional testing is needed to examine whether such methodologies would prove beneficial in the operational setting. Additionally, while much improved over the standard "wet sponges," the electrode technology needs further refinement making them less obtrusive and more comfortable in human operators.

\section{References}

Ahlberg, J., Klasen, L., Gronwall, C., Ulvklo, M., \& Jungert, E. (2003). Automatic target recognition on a multisensor platform. In Proceedings of the Swedish Symposium on Image Analysis, 93-96.

Ambrus, G. G., Antal, A., \& Paulus, W. (2011). Comparing cutaneous perception induced by electrical stimulation using rectangular and round shaped electrodes. Clinical Neurophysiology, 122, 803-7. doi:10.1016/ j.clinph.2010.08.023

Antal, A., Nitsche, M. A., Kinsces, T. Z., Kruse, W., Hoffmann, K.-P., \& Paulus, W. (2004). Facilitation of visuomotor learning by transcranial direct current stimulation of the motor and extrastriate visual areas in humans. European Journal of Neuroscience, 19, 2888-2892. doi: 10.1111/j.1460-9568.2004.03367.x

Bangera, N. B. (2008). Development and validation of a realistic head model for EEG (Doctoral thesis).

Available from ProQuest Dissertations and Theses database. (Publication No: AAI3308124).

Bangera, N. B., Schomer, D. L., Dehghani, N., Ulbert, I., Cash, S., Papavasiliou, S., . . Halgren, E. (2010). 
Experimental validation of the influence of white matter anisotropy on the intracranial EEG forward solution. Journal of Computational Neuroscience, 29, 371-387. doi: 10.1007/s10827-009-0205-z

Berryhill, M. E., \& Jones, K. T. (2012). tDCS selectively improves working memory in older adults with more education. Neuroscience Letters, 521, 148-151. doi:10.1016/j.neulet.2012.05.074

Bolognini, N., Fregni, F., Casati, C., Olgiati, E., \& Vallar, G. (2010). Brain polarization of parietal cortex augments training-induced improvement of visual exploratory and attentional skills. Brain Research, 1349, 76-89. doi:10.1016/j.brainres.2010.06.053

Bullard, L. M., Browning, E. S., Clark, V. P., Coffman, B. A., Garcia, C. M., Jung, R. E., . . W Weisend, M. P. (2011). Transcranial direct current stimulation's effect on novice versus experienced learning. Experimental Brain Research, 213, 9-14. doi:10.1007/s00221-011-2764-2 Carlson, N. R. (2010). Physiology of behavior (10th ed.). Boston, MA: Allyn \& Bacon.

Chi, R. P., Fregni, F., \& Snyder, A. W. (2010). Visual memory improved by non-invasive brain stimulation. Brain Research, 1353, 168-175. doi:10.1016/j.brainres.2010.07.062

Chi, R. P., \& Snyder, A. W. (2011). Facilitate insight by non-invasive brain stimulation. PloS one, 6(2), e16655. doi:10.1371/journal.pone.0016655

Chief Scientist (Air Force) Washington, D. C. (2010). Report on technology horizons: A vision for Air Force science and technology during 2010-2030 (Vol. 1, Air Force Tech. Rep. No. AF/ST-TR-10-01). Washington, DC: Department of the Air Force.

Clark, V. P., Coffman, B. A., Mayer, A. R., Weisend, M. P., Lane, T. D. R., Calhous, V. D., . . Wassermann, E. M. (2012). TDCS guided using fMRI significantly accelerates learning to identify concealed objects.

Neurolmage, 59, 117-128. doi:10.1016/j.neuroimage.2010.11.036

Coffman, B. A., Trumbo, M. C., Flores, R. A., Garcia, C. M., van der Merwe, A. J., Wassermann, E. M., . . . Clark, V. P. (2012). Impact of tDCS on performance and learning of target detection: Interaction with stimulus characteristics and experimental design. Neuropsychologia, 50(7), 1594-1602.

doi:10.1016/j.neuropsychologia.2012.03.012

Dale, A. M., Fischl, B., \& Sereno, M. I. (1999). Cortical surface-based analysis I: Segmentation and surface reconstruction. Neurolmage, 9, 179-194. doi:10.1006/nimg.1998.0395

Dassault-Systèmes. (2007). ABAQUS overview. Retrieved from http://www.3ds.com/productsservices/simulia/portfolio/abaqus/overview/

Datta, A., Bansal, V., Diaz, J., Patel, J., Reato, D., \& Bikson, M. (2009). Gyri-precise head model of transcranial DC stimulation: Improved spatial focality using a ring electrode versus conventional rectangular pad. Brain Stimulation, 2, 201-207. doi:10.1016/j.brs.2009.03.005

Desimone, R. (1996). Neural mechanisms for visual memory and their role in attention. PNAS, 93, 1349413499. doi:10.1073/pnas.93.24.13494

Ditye, T., Jacobson, L., Walsh, V., \& Lavidor, M. (2012). Modulating behavioral inhibition by tDCS combined with cognitive training. Experimental Brain Research, 219, 363-368. doi:10.1007/s00221-012-3098-4

Elmer, S., Burkard, M., Renz, B., Meyer, M., \& Jancke, L. (2009). Direct current induced short-term modulation of the left dorsolateral prefrontal cortex while learning auditory presented nouns. Behavioral and Brain Functions: BBF, 5, 29. doi:10.1186/1744-9081-5-29

Falcone, B., Coffman, B. A., Clark, V. P., \& Parasuraman, R. (2012). Transcranial direct current stimulation augments perceptual sensitivity and 24-hour retention in a complex threat detection task. PLoS One, 7(4), e34993. doi:10.1371/journal.pone.0034993

Fecteau, S., Knock, D., Fregni, F., Sultani, N., Boggio, P. S., \& Pascual- Leone, A. (2007). Diminishing risk-taking behavior by modulating activity in the prefrontal cortex: A direct current stimulation study. The Journal of Neuroscience, 27, 12500-12505. doi:10.1523/JNEUROSCI.3283-07.2007

Fecteau, S., Pascual-Leone, A., Zald, D. H., Liguoir, P., Théoret, H., Boggio, P. S., \& Fregni, F. (2007). Activation of prefrontal cortex by transcranial direct current stimulation reduces appetite for risk during ambiguous decision making. The Journal of Neuroscience, 27, 6212-6218. doi:10.1523/JNEUROSCI.0314-07.2007

Fischl, B., Salat, D., Busa, E., Albert, M., Dieterich, M., Haselgrove, C., . . . Dale, A. M. (2002). Whole brain 
segmentation: Automated labeling of neuroanatomical structures in the human brain. Neuron, 33, 341-355. doi:10.1016/S0896-6273(02)00569-X

Flöel, A., Rösser, N., Michka, O., Knecht, S., \& Breitenstein, C. (2008). Noninvasive brain stimulation improves language learning. Journal of Cognitive Neuroscience, 20, 1415-1422. doi:10.1162/jocn.2008.20098

Fregni, F., Boggio, P. S., Nitsche, M., Bermpohl, F., Antal, A., Feredoes, E., . . . Pascual-Leone, A. (2005). Anodal transcranial direct current stimulation of prefrontal cortex enhances working memory.

Experimental Brain Research, 166, 23-30. doi:10.1007/s00221-005-2334-6

Fritsch, B., Reis, J., Martinowich, K., Schambra, H. M., Ji, Y., Cohen, L. G., \& Lu, B. (2010). Direct current stimulation promotes BDNF- dependent synaptic plasticity: Potential implications for motor learning. Neuron, 66, 198-204. doi:10.1016/j.neuron.2010.03.035

Gladwin, T. E., den Uyl, T. E., Fregni, F. F., \& Wiers, R. W. (2012a). Enhancement of selective attention by tDCS: Interaction with interference in a Sternberg task. Neuroscience Letters, 512, 33-37. doi:10.1016/ j.neulet.2012.01.056

Gladwin, T. E., den Uyl, T. E., \& Wiers, R. W. (2012b). Anodal tDCS of dorsolateral prefontal cortex during an Implicit Association Test. Neu-roscience Letters, 517, 82-86. doi:10.1016/j.neulet.2012.04.025

Hammer, A., Mohammadi, B., Schmicker, M., Saliger, S., \& Munte, T. F. (2011). Errorless and errorful learning modulated by transcranial direct current stimulation. BMC Neuroscience, 12, 72. doi: 10.1186/1471-2202-1272

Hecht, D., Walsh, V., \& Lavidor, M. (2010). Transcranial direct current stimulation facilitates decision making in a probabilistic guessing task. The Journal of Neuroscience, 30, 4241-4245. doi:10.1523/JNEUROSCI .2924-09.2010

Kincses, T. Z., Antal, A., Nitsche, M. A., Bártfai, O., \& Paulus, W. (2004). Facilitation of probabilistic classification learning by transcranial direct current stimulation of the prefrontal cortex in the human. Neuropsychologia, 42, 113-117. doi:10.1016/S0028-3932(03)00124-6

Márquez-Ruiz, J., Leal-Campanario, R., Sanchez-Campusano, R., Molaee- Ardekani, B., Wendling, F., Miranda, P. C., . . . Dalgado-Garcia, J. M. (2012). Transcranial direct-current stimulation modulates synaptic mechanisms involved in associative learning in behaving rabbits. PNAS, 109, 6710-6715.

doi:10.1073/pnas.1121147109

McKinley, R. A., Bridges, N., Walters, C. M., \& Nelson, J. (2012). Modulating the brain at work using noninvasive transcranial stimulation. Neurolmage, 59, 129-137. doi:10.1016/j.neuroimage.2011.07.075

McKinley, R. A., Nelson, J. T., \& McIntire, L. K. (2010, October). Monitoring hemodynamic changes caused by tDCS. Paper presented at the Brain Stimulation Colloquium, Albuquerque, NM.

Mirenowicz, J., \& Schultz, W. (1994). Importance of unpredictability for reward responses in primate dopamine neurons. Journal of Neurophysiology, 72, 1024-1027.

Mirenowicz, J., \& Schultz, W. (1996). Preferential activation of mid-brain dopamine neurons by appetitive rather than aversive stimuli. Nature, 379, 449-451. doi:10.1038/379449a0

Mishra, A. K., \& Mulgrew, B. (2010). Automatic target recognition. In R. Blockley \& W. Shyy (Eds.), Encyclopedia of aerospace engineering. Hoboken, NJ: Wiley.

Mulquiney, P. G., Hoy, K. E., Daskalakis, Z. J., \& Fitzgerald, P. B. (2011). Improving working memory: Exploring the effect of transcranial random noise stimulation and transcranial direct current stimulation on the dorsolateral prefrontal cortex. Clinical Neurophysiology, 122, 2384-2389. doi:10.1016/j.clinph.2011.05.009

Nelson, J. T. (2007). Enhancing warfighter cognitive abilities with transcranial magnetic stimulation: $A$ feasibility analysis (U.S. Air Force Tech. Rep. No. AFRL-HE-WP-TR-2007-0095). Washington, DC: Department of the Air Force.

Nelson, J. T., McKinley, R. A., Golob, E. J., \& Warm, J. S. (2013). Enhancing vigilance in operators with prefrontal cortex transcranial direct current stimulation (tDCS). Neurolmage. Advance online publication. doi:10.1016/j.neuroimage2012.11.061

Nitsche, M. A., Fricke, K., Henschke, U., Schlitterlau, A., Liebetanz, D., Lang, N.,... Paulus, W. (2003). Pharmacological modulation of cortical excitability shifts induced by transcranial direct current stimulation 
in humans. Journal of Physiology, 553, 293-301. doi:10.1113/jphysiol.2003.049916

Paulus, W. (2003). Transcranial direct current stimulation (tDCS). Supplements to Clinical Neurophysiology, 56, 249-254. doi:10.1016/S1567- 424X(09)70229-6

Penolazzi, B., Di Domenico, A., Marzoli, D., Mammarella, N., Fairfield, B., Franciotti, R., . . Tommasi, L. (2010). Effects of transcranial direct current stimulation on episodic memory related to emotional visual stimuli. PloS One, 5(5), e10623. doi:10.1371/journal.pone.0010623

Petree, L. E., Bullard, L. M., Jung, R. E., Shoemaker, J. M., Vakhtin, A. A., van der Merwe, A. J., \& Weisend, M. P. (2011, November). Alternative electrode methodology for the administration of transcranial direct current stimulation. Paper presented at the Society for Neuroscience Annual Meeting, Washington, DC.

Priori, A. (2003). Brain polarization in humans: A reappraisal of an old tool for prolonged non-invasive modulation of brain excitability. Clinical Neurophysiology, 114, 589-595. doi:10.1016/S13882457(02)00437-6

Reis, J., Schambra, H. M., Cohen, L. G., Buch, E. R., Fritsch, B., Zarahn, E., . . . Krakauer, J. W. (2009). Noninvasive cortical stimulation enhances motor skill acquisition over multiple days through an effect on consolidation. PNAS, 106, 1590-1595. doi:10.1073/pnas.0805413106

Ross, L. A., McCoy, D., Wolk, D. A., Coslett, H. B., \& Olson, I. R. (2010). Improved proper name recall by electrical stimulation of the anterior temporal lobes. Neuropsychologia, 48, 3671-3674. doi:10.1016/j .neuropsychologia.2010.07.024

Sadleir, R. J., Vannorsdall, T., Schretlen, D., \& Gordon, B. (2010). Predicted current densities in transcranial direct current stimulation in a realistic head model. Neurolmage, 51, 1310-1318.

doi:10.1016/j.neuroimage.2010.03.052

Scott, K. (2012, February 7). Neuroscientists to top brass: Mess with minds. . carefully. Wired. Retrieved from http://www.wired.com/dangerroom/2012/02/neuroscience-war/

Sorkin, R. D. (1999). Spreadsheet signal detection. Behavior Research Methods, Instruments \& Computers, 31, $46-54$. doi:10.3758/BF03207691

Sparing, R., Dafotakis, M., Meister, I. G., Thirugnanasambandam, N., \& Fink, G. R. (2008). Enhancing language performance with non-invasive brain stimulation-A transcranial direct current stimulation study in healthy humans. Neuropsychologia, 46, 261-268. doi:10.1016/j.neuropsychologia.2007.07.009

Tanji, J., \& Hoshi, E. (2008). Role of the lateral prefrontal cortex in executive behavioral control. Physiological Reviews, 88, 37-57. doi: 10.1152/physrev.00014.2007

Teo, F., Hoy, K. E., Daskalakis, Z. J., \& Fitzgerald, P. B. (2011). Investigating the role of current strength in tDCS modulation of working memory performance in healthy controls. Frontiers in Psychiatry, 2, 45. doi:10.3389/fpsyt.2011.00045

Visualization Sciences Group. (2007). AMIRA-Advanced 3D visualization and volume modeling (Software). Burlington, MA: Author.

Wagner, T., Valero-Cabre, A., \& Pascual-Leone, A. (2007). Noninvasive human brain stimulation. Annual Review of Biomedical Engineering, 9, 527-565. 